\title{
How to Avoid Conceptualization and Formalization of Anti-corruption in Peking Opera
}

\author{
Yifan Xue* \\ Linyi University of Music, Linyi, China \\ *Corresponding author

\begin{abstract}
This article discusses the problems encountered in the creation of the popular anti-corruption theme. Taking the Beijing opera "On the Road" as an entry point, it analyzes the concept, creative direction, composition, and plot of the drama. "On the Road" is created with people as the center and is grounded to avoid such dramas. The missionary intention is obviously embarrassing. The essential elements of other dramas are also very high. The script is good, the music is beautiful, the actors are great, and many others. The elements are complete, which contributed to the good show that resonated with the audience and penetrated into the hearts of the people.
\end{abstract} \\ ABSTRACT
}

Keywords: Anti-corruption play, Peking opera, "On the Road", Conceptualization, Formalization

\section{京剧反腐戏如何避免概念化、形式化 \\ 薛一凡*}

临沂大学音乐学院, 临沂, 中国

“通讯作者

\section{中文摘要}

本文对当下大热的反腐题材创作中遇到的问题进行探讨，以京剧《在路上》为切入点，分析了该剧的 立意、创作导向、作曲、戏剧情节等方面，《在路上》是以人民为中心创作，接地气，避免了此类戏 人物不接地气, 宣教意图明显的尴尬, 其他戏曲必须的元素水准亦很高, 剧本好, 音乐美, 演员棒, 诸多元素齐备, 促成了这部引起观众共鸣的好戏且深入人心。

关键词: 反腐戏; 京剧; 在路上; 概念化; 形式化

京剧《在路上》是一部现代反腐戏,一首演就被冠以 京剧版《人民的名义》 1)，也算“蹭”了大热的 IP 《人民 的名义》, 热度想要蹭的好, 主创团队们要有自信和胆 量, 很容易被比较, 比较便会有高下, 但是《在路上》 首演以来, 受到了各界追捧, 主演万晓慧, 王小蝉带该 作登上了“2018 年新年戏曲晚会”, 中央党校官网、新华 网、光明日报、央广网等知名网媒、报媒对其发表评论, 赞叹之声不绝, 并且在湖北省进行了巡演, 可见该剧本 身热度不低。可见该剧深受观众接受和欢迎, 从受众角 度来看, 也渴望有能真正反映官场现实和揭露官场黑暗 的好戏, 但艺术作品中能真正敢说敢写敢演的却是风毛 麟角。现代题材的反腐剧“度”不好把握, 太容易概念化、 形式化, 现代戏本来就有自带的属性, 被诟病不像京剧, 话剧加唱等等。要演反腐题材的更加难以把握, 如何定 位, 如何演老百姓买帐, 不做成党的宣传片和宣教片。
最终要做成艺术品, 就得在艺术性上着重塑造。

这部反腐戏来说，完整真实的展现了反贪反腐背后 的故事。首先是以人民为中心创作, 接地气, 避免了此 类戏人物不接地气，宣教意味浓厚的的尴尤。《在路上》 具有鲜明的思想教育目的和功能。那么就是这样一台戏 为什么还能引起共鸣, 其一是因为当下反腐深入人心, 再者剧本好, 音乐美, 演员棒, 也是成为一台好戏的必 备条件。《在路上》是以人民为中心为创作导向，选材 是从人命案入手牵扯出贪腐案, 增强了戏剧冲突。人物 设定上避免了概念化, 剧中的韩梅首先具备一个党员干 部应有的操守, 而从人性化的角度来看, 首先她是一个 女人，她又是个爱生活、爱孩子的女人，她的内心情感 丰富细滅。作为一个女纪检干部，如何表现她的铁骨柔 情，所以她内心戏挺多，让人物更真实、丰满，避免符 号化。这出戏挖掘了一些让人感动的细节比如给李大婶 
喂药, 涉夜不眠的工作, 还有反映官场黑暗的唱词, “我 冤也喊了, 状也告了, 可他们有钱有势, 没人敢帮, 上 边交代调查组定了案已经是铁板钉钉的事, 不准再提, 再要提连我那当村主任侄儿也要遭殊”很多反映官商勾 结, 仗势欺人的唱词都博得观众阵阵掌声, 因为你说出 了观众所想说的话。因为这种深入的挖掘, 从而展现人 性的深度和复杂性, 这样更能打动观众, 让整部戏更有 思想性和张力。

音乐为整部剧增色不少，作曲家创作手法灵活多 变, 在刻画人物情感上颇为有力。目前的唱腔创作大体 处于择腔安调, 导致在情感表达上略显不足, 无法深层 次刻画人物情感。而《在路上》的音乐创作很有张力。 这部戏的声腔布局、唱段设计上, 运用了不同的声腔板 式体现不同的情绪, 活用了京剧传统元素。这出戏使用 了西皮腔、二黄腔、反二黄腔、南梆子、汉调、吹腔、 娃娃调、昆曲等, 核心唱段使用了反二黄、二黄腔, 作 曲家对这些声腔运用自如, 足见其功底之深厚。如第七 场, 母子争吵从而反目的戏, 用了一段【反二黄慢板】, 母亲因难言之隐无法告知女儿详情, 而女儿也不理解 她, 母亲纠结、痛苦的感情通过这段唱腔表达的淋漓尽 致。这段腔的设计极具表现力, 中低音区低回深沉, 高 音区悲愤激励、苍凉凄楚。第九场百姓前来看望韩梅一 场, 最后将剧推向高潮的一场, 使用了娃娃调来表现韩 梅的觉悟以及爱党爱民的形象, “这段腔的旋律起伏大、 高六明亮、棱角突出、钢劲挺拔(2)。”其中【原板】的 唱词字字珠现, “你给百姓一粒种, 万斛粮仓堆起来。 你给百姓一滴水, 万顷清波涌出来。你给百姓一捧土, 万仞高山垒起来。你给百姓一片云, 万朵彩霞升起 来......”。

为了增加音乐性还加入了伴唱和二重唱的形式, 特 别是二重唱的唱词是把赵市长曾经的初心、理想信念用 了“你也曾”的排比句来展现，“你也曾昂首阔步超前奔 跑, 你也曾把准航向定目标, 你也曾尽心竭力把工作做 好, 你也曾为党为民立功劳......”生一旦两个音乐织 体交相呼应, 增强叙事性, 现场效果颇佳。该戏的另一 特色采用了大量的对唱, 赵市长和孙总的对唱, 老夫妻 俩的对唱, 赵市长和韩梅的对唱, 每段对唱都有自己鲜 明的特色, 很有力度, 听起来也很过瘾。除了主演万晓 慧精彩表演外, 不得不提李大婶, 老旦李大婶的唱腔、 表演着实让人眼前一亮, 作为故事主线人命案的直接受 害者, 李大婶和李大叔老两口是整出戏矛盾的引子, 亦 是整部戏的支点, 支点支的准确到位能尧起的重量就越 大, 这段戏演的好为矛盾冲突起到了推波助澜作用。演 员表演极具张力, 唱腔铿铭有力, 念白字字剜心, 那种 悲痛的气氛, 观者无不为之动容, 实在是为整出戏增色 的一笔。

舞美虚实结合, 风格清新、洗练、空灵、流畅, 充 分运用了几何图形, 很具生活气息和现代感。灯光切换 流畅, 亦与时空切换结合紧密。

在演员表演上, 用了话剧的表演手法, 形体、语言 更贴近现代生活状态。

这部戏虽然做了一定的突破, 但概念化、形式化的 内容还是有几处体现。还是应摒弃形式化、口号化的表
演, 通过故事、表演由内而外体现，这种表演才不会招 致反感。艺术创作要有生活逻辑和常识, 有几处逻辑似 有悖常理。

一是两个孩子领证结婚, 领证手续大家都知道要身 份证和户口本, 而该戏一直围绕身份证作文章, 没有提 户口本的事, 是否有欠缺呢。

二是赵市长入狱, 他儿子赵翔没有任何纠结就继续 要跟陈红结婚, 赵翔缺了心理斗争的戏份, 过渡不甚流 畅。

三是乡亲们听说韩梅要调走来看她, 不希望青天韩 梅走, 而韩梅说离不开江城, 不走了。就现在官员升迁 调动制度来说不现实，一般政府官员在一地任职时间不 会太久应服从组织安排。这也反映出另一个问题是人民 的青天情结和制度建设, 希望有青天来拯救自己, 而不 是呼唤进行制度完善。中国现在是法治社会, 完善制度 才是保证公平、正义的根本途径, 所以作为一部现代反 腐戏, 应该引领正确的价值取向, 该戏在结尾不应停留 在呼唤清官, 留住青天的层面。应体现目前国家在完善 制度，让人们放心有制度保障，即使她走了，也不用担 心。这种设置比较符合现在的国情。青天情节可以用, 但是现代戏中对青天的使用要有度。

四是是否可以增强反派角色, 在揭露的是官商勾结 和无良商人时应该加大矛盾冲突, 把对手强大起来, 这 样的对手戏才更好看, 所谓魔高一尺道高一丈, 这样整 部戏的可看性就增强了。

五是乡亲们听说韩梅要调走来看她这场有点偏形 式化, 最怕看到的就是有高度政治化的样板戏的痕迹。 第八场韩梅劝说赵市长一场, 偏形式化, 且前后情节逻 辑上有点问题, 缺乏可行性, 不知是否是为了表现这句 “不忘初心, 方得始终”而刻意为之。照理说赵市长是非 常恨韩梅, 断送了他的仕途, 但在韩梅简单劝说之后就 欣然接受了。并且劝说的过程是从党员的觉悟上入手, 从入党的初衰到入党誓词, 瞬间把人物拔高了, 拉大了 跟观众的距离感。劝说应该更人性化, 从他俩的老同学 关系, 儿女结亲的这些层面劝说是否更合理一些, 赵市 长在是党员之前, 他首先是个人。

\section{REFERENCES}

[1] Yingxian Cheng, Lan Ye Chun Wei, Xin Xin Ci Sheng Yi -- Comments on watching the 8th Beijing Opera Festival, Art Review, 07(2017)14-23

[2] Lianzhong Zhi, Where Does A Good Play Popular with the Common People Come from, Chinese Arts News, 003(2019)

[3] Zhi Zhang, Qing Pin Nai De Shi Zuo Guan Xiu De Li Zhi Xian Shou Lian -- Beijing Opera < Chen Tingjing > Take Part in the 8th Beijing Opera Festival, XiYou, 02(2017) 62-63+75 
[4] Kui AN, Reflections on Peking Opera after the Festival--On the 8th Beijing Opera Festival, Art Review, 07(2017)7-13.

[5] Hao Liu, Jiu Shi Jin Fen Di Jin Zhao Pi Huang Yang -- The Eighth Beijing Opera Festival, Jingju of China, 07(2017)10-15+2+97. 